\title{
USO SEGURO DE LOS MEDICAMENTOS EN ADULTOS MAYORES: UNA LISTA DE CHEQUEO
}

\author{
Teodoro J. Oscanoa ${ }^{1,2, a}$
}

\begin{abstract}
RESUMEN
Los adultos mayores son más propensos a presentar reacciones adversas medicamentosas (RAM), debido a la polifarmacia que caracteriza a aquellos con múltiples enfermedades, y cambios, que por la edad ocurren en la farmacocinética y la farmacodinamia de los medicamentos. La evaluación de los aspectos de seguridad y uso racional de los medicamentos son importantes en esta población; por ello, se propone una lista de chequeo que consiste en hacer una lista de medicamentos (incluye la medicina herbaria); detectar e intervenir síndromes geriátricos inducidos por medicamentos, sobreprescripción, disprescripción e infraprescripción; medir e intervenir la adherencia farmacológica, medir los parámetros para posología geriátrica; prevenir las reacciones adversas por retiro inadecuado de fármacos, evaluar la capacidad de manejo de medicamentos de la persona mayor y utilizar la hoja de información mínima sobre el fármaco prescrito al paciente. Esta lista de chequeo construida en base a instrumentos validados, es una propuesta cuya aplicación en el contexto ambulatorio y hospitalario es posible y factible.
\end{abstract}

Palabras clave: Prescripciones de medicamentos; Evaluación de medicamentos; Anciano frágil (fuente: DeCS BIREME).

\section{SAFE USE OF MEDICATIONS AMONG ELDERLY PEOPLE: A CHECKLIST}

\section{RESUMEN}

Elderly people are particularly vulnerable to adverse drug reactions (ADR) due to polypathology and polypharmacy and the changes in pharmacokinetics and pharmacodynamics of medications induced by aging. It is very important to evaluate the safety aspects and appropriate use of medications in this population. For this purpose, a checklist is proposed consisting of a list of medications (including herbal medicine), detecting and treating geriatric syndromes induced by medications, overprescription, unprescription and underprescription; measuring and treating drug adhesion, measuring parameters for geriatric posology, preventing adverse reactions due to inadequate drug recalls, evaluating aging people's capacity to take their medications and using the minimum datasheet regarding the medication prescribed to the patient. This checklist is developed based on validated instruments. It is a proposal which application in the outpatient and inpatient context is possible and feasible.

Key words: Drug prescriptions; Drug evaluation; Frail elderly (source: MeSH NLM).

\section{INTRODUCCIÓN}

En 1999 el Instituto de Medicina (IOM) una de las instituciones más respetadas de EE. UU. publicó el libro: "Errar es humano", donde se revelaba que cerca de 98000 personas morían anualmente como resultado de errores médicos, e instaba a instituciones sanitarias a juntar esfuerzos para construir un sistema de salud seguro (1).
Los eventos y reacciones adversas medicamentosas (RAM) están entre las causas principales de morbimortalidad. Según una reciente revisión sistemática, las personas mayores son las que presentan elevadas prevalencias de RAM en el contexto ambulatorio $(16,1 \%)$ comparado con el $2.5 \%$ en niños y $5,3 \%$ en adultos jóvenes ${ }^{(2)}$.

En otro estudio sobre las atenciones en los departamentos de emergencia debido a RAM, el 37,5\%

\footnotetext{
Centro de Investigación del Envejecimiento, Facultad de Medicina, Universidad San Martín de Porres. Lima, Perú.

Hospital Nacional Guillermo Almenara Irigoyen. Lima, Perú.

Médico especialista en medicina interna, doctor en Medicina.

Recibido: 06-12-12 Aprobado: 17-04-13
}

Citar como: Oscanoa TJ. Uso seguro de los medicamentos en adultos mayores: una lista de chequeo. Rev Peru Med Exp Salud Publica. 2013;30(2):320-5. 
requirió hospitalización, la mayoría de las RAM fueron catalogados como potencialmente prevenibles; los fármacos implicados en la mayor parte de las reacciones adversas fueron warfarina, insulinas, antiplaquetarios orales e hipoglicemientes orales ${ }^{(3)}$.

Estudios fármaco-económicos de los denominados problemas relacionados con medicamentos arrojan costos impresionantes: 76 mil millones de dólares en pacientes ambulatorios, 20 mil millones de dólares en hospitalizaciones y 4 mil millones de dólares en residencias de ancianos ${ }^{(4)}$. Un estudio realizado en Lima Perú, encontró que el $24 \%$ de las hospitalizaciones en personas mayores fueron causados por RAM ${ }^{(5)}$.

El uso seguro de los medicamentos en adultos mayores requiere la prevención de tres principales errores de prescripción farmacológicas: sobreprescripción, disprescripción e infraprescripción de fármacos. Sobreprescripción es prescribir más medicamentos que los clínicamente necesarios ${ }^{(6)}$. Disprescripción es la prescripción incorrecta de un fármaco que tiene una indicación clínica, los errores pueden ser selección del fármaco, dosis, forma de administración, duración de la terapia, interacción medicamentosa, monitorización y costo ${ }^{(6)}$. La infraprescripción es la omisión de una intervención farmacológica de probada efectividad según criterios de medicina basada en evidencias, existiendo la condición o enfermedad que exigía tal intervención terapéutica ${ }^{(7)}$.

La prevalencia de subprescripción farmacológica en personas mayores es de $42 \%$, entre los cuales figuran omisión de prescripción de medicamentos, existiendo la indicación para estatinas, antiplaquetarios, metformina, inhibidores de la enzima convertidora de angiotensina para falla cardiaca crónica y aspirina o clopidogrel para historia de cardiopatía coronaria o enfermedad vascular periférica ${ }^{(8)}$.

Actualmente, uno de los instrumentos más utilizados sobre prescripción potencialmente inadecuada de medicamentos en adultos mayores son los criterios de Beers ${ }^{(9)}$, cuya última versión fue publicada el $2012{ }^{(10)}$. Uno de los objetivos principales de los criterios de Beers fue la de servir como un instrumento para la prevención de eventos adversos causados por medicamentos, sobre la hipótesis que los fármacos incluidos en dicha lista estaban asociados a resultados adversos en la población geriátrica que lo consumía.

En una revisión sistemática sobre la asociación de resultados sobre la salud asociados a los criterios de Beers, no se encontró asociación con mayor mortalidad y otros resultados (Ejem. hospitalización, deterioro funcional, etc.) los efectos sobre calidad de vida y costos no fueron concluyentes; sobre las RAM solo se incluyó tres estudios, dos de las cuales mostraron asociación significativa (11). Por consiguiente, se hace necesario buscar estrategias diferentes de las listas explicitas (Ejem. criterios de Beers), con el objetivo de hacer un uso racional y seguro de los medicamentos en personas mayores.

El presente trabajo es una propuesta de una lista de chequeo para el uso seguro de los medicamentos en personas mayores, con el fin de prevenir la sobreprescripción, disprescripción y la infraprescripción de medicamentos en esta población, los aspectos de seguridad de la medicación también incluyen la prevención de problemas relacionados con medicamentos (RAM, interacciones farmacológicas, adherencia, etc.). Esta lista de chequeo consta de diez pasos, cada uno de ellos están basados en instrumentos y estrategias validadas, detallándose en los casos que se requiera, sus propiedades clinimétricas (sensibilidad, especificidad u otros detalles estadísticos de confiabilidad).

\section{USO SEGURO DE LOS MEDICAMENTOS EN PERSONAS MAYORES: UNA LISTA DE CHEQUEO}

\section{PASO 1. HACER UNA LISTA DE MEDICAMENTOS TOMADOS POR EL PACIENTE}

La mejor estrategia para tener una lista completa de medicamentos que toma el paciente es solicitar al adulto mayor o al cuidador que traiga al consultorio todos los medicamentos prescritos por médicos, otros profesionales o los automedicados (Brown bag test) ${ }^{(12)}$, debe insistirse en que estos incluyen las vitaminas, gotas oculares, estén usándose o no. Los medicamentos con fecha de vencimiento caducados que se identifiquen deben ser eliminados.

\section{PASO 2. HACER UNA LISTA DE MEDICINA HERBARIA DEL PACIENTE}

El consumo de suplementos herbarios se ha extendido de manera vertiginosa, especialmente en adultos mayores tanto en países desarrollados como subdesarrollados. Los aspectos de eficacia y especialmente de seguridad de estos productos en las personas mayores son un tema pendiente de investigación, por consiguiente es sumamente importante la vigilancia y observaciones de los pocos datos que tienen al respecto en la actualidad. Existe un grado importante de desinformación sobre los riesgos de la medicina herbaria ${ }^{(13)}$ 


\section{PASO 3. DETECCIÓN DE SÍNDROMES GERIÁTRICOS INDUCIDOS POR MEDICAMENTOS}

Los principales grupos farmacológicos asociados con síndromes de caídas en ancianos son las benzodiacepinas, antidepresivos y antipsicóticos (14). Por otro lado, el enfoque preventivo del síndrome de delirium y el trastorno cognitivo inducido por medicamentos, está dirigido fundamentalmente al uso racional de medicamentos con carga anticolinérgica. Entre los instrumentos más útiles y prácticos, que pueden evidenciar una alta carga anticolinérgica y de sedantes están: la escala de riesgo anticolinérgico (Anticholinergic Risk Scale) ${ }^{(15)}$, y el índice de carga de medicamentos (ICM o Drug Burden Index) ${ }^{(16) .}$

\section{PASO 4. DETECTAR E INTERVENIR SOBRE LA SOBREPRESCRIPCIÓN Y DISPRESCRIPCIÓN}

El uso apropiado de medicamentos (Medication Appropriateness Index o MAI) ${ }^{(17)}$ es un instrumento descrito por Hanlon et al. y está diseñado para evaluar la calidad de la prescripción farmacológica en adultos mayores, especialmente lo referente a problemas catalogados como de sobreprescripción y disprescripción.

El desarrollo del MAl consiste en la calificación de las siguientes características principales de una prescripción: indicación, efectividad, dosis, instrucciones correctas, instrucciones prácticas, interacción farmacológica, interacción fármaco-enfermedad, redundancia innecesaria, duración, y costo. Puede considerarse que el desarrollo del MAl es fundamentalmente una auditoría de la prescripción farmacológica, teniendo como referencia las guías de práctica clínica actualizadas sobre la farmacoterapia específica de cada caso evaluado.

Las propiedades clinimétricas de este instrumento reflejan su utilidad ${ }^{(17)}$, la confiabilidad intertasador total para señalar lo apropiado de un medicamento fue de 0,88 , y para señalar lo inadecuado de una prescripción medicamentosa fue de 0,95 para un valor kappa total de 0,83 . Por otro lado, la confiabilidad intratasador para la calificación de lo adecuado de una prescripción medicamentosa fue de 0,94 y para señalar lo inapropiado de una medicación específica fue de 0,98 para un valor kappa total de 0,92.

\section{PASO 5. MEDICIÓN E INTERVENCIÓN SOBRE ADHERENCIA FARMACOLÓGICA}

El grado de adherencia farmacológica es la medida en que el paciente asume las normas o consejos dados por el médico o personal sanitario, tanto desde el punto de vista de hábitos o estilo de vida recomendados, como del propio tratamiento farmacológico prescrito, expresándose el grado de coincidencia entre las orientaciones dadas por el profesional y la realización por parte del paciente, realizadas estas tras una decisión completamente razonada, por este, abandonándose las connotaciones de sumisión que este término implicaba para el paciente ${ }^{(18)}$.

Debe recordarse que "los fármacos no funcionan en los paciente que no los toman" (19), por consiguiente debe medirse al grado de adherencia al tratamiento, dado que la mayoría de fracasos es por incumplimiento de la terapia por parte del paciente. Existen varias formas de detectar no-adherencia, la más útil es el cuestionario de Morisky ${ }^{(20)}$, (sensibilidad de $72,2 \%$ y especificidad del $74,1 \%$ ); una vez detectada la no adherencia, debe establecerse la causa e instaurar la mejor medida de intervención.

Las estrategias para mejorar la adherencia a la terapia farmacológica, especialmente en pacientes polimedicados de probada efectividad en pacientes adultos mayores (19), son las siguientes: educación sobre el medicamento, instrucciones escritas (Paso 10), simplificación del régimen terapéutico, educación sobre la enfermedad, sesiones o consultas de revisión de medicamentos, uso de organizadores de medicamentos, instrucciones escritas sucintas (Ejem. hojas plastificada o laminada sobre los medicamentos del paciente y sus instrucciones), y calendarios donde se especifican el día y la hora de la toma de medicamentos.

\section{PASO 6. MEDIR PARÁMETROS PARA POSOLOGÍA GERIÁTRICA: PESO, TALLA Y DEPURACIÓN RENAL CALCULADA}

Administrar la dosis exacta para un paciente específico es una de las principales medidas de prevención de las RAM. Para el cálculo de la posología es necesario tener parámetros como el peso, talla y la depuración estimada de creatinina, especialmente para los fármacos que son eliminados por vía renal.

Un reciente metanálisis ${ }^{(21)}$, comparó la exactitud de las fórmulas de estimación de tasa de filtración glomerular con métodos de referencia (Vgr inulina, Cr-51-EDTA, Tc-DTPA o iohexol), la fórmula de Cockcroft-Gault (22), MDRD (Modification of Diet in Renal Disease) (23) y concentración sérica de cistatina $C$ mostraron ser más cercanos al patrón de referencia. En orden decreciente en sensibilidad las pruebas para medir la función renal en adultos mayores según el estudio, son: CockcroftGault ${ }^{(22)}$ y MDRD ${ }^{23)}$ (80 - 100\% ambos); concentración de cistatina C (86 - 97\%); depuración de creatina en orina de 24 h (64-93\%), y creatinina sérica (40 - 60\%) ${ }^{(21)}$. De acuerdo al estudio, es posible usar indistintamente 
la fórmula de Cockcroft-Gault y MDRD. Puede seleccionarse la fórmula MDRD en los casos en que no se disponga del peso o no se requiera la corrección por área de superficie corporal (en esta fórmula no se requiere la talla del paciente); son útiles tanto la fórmula MDRD o su forma abreviada, esta última fórmula tiene la ventaja de ser más exacta y precisa que la de CockcroftGault cuando la tasa de filtración glomerular es menor de $60 \mathrm{~mL} / \mathrm{min} / 1,73 \mathrm{~m}^{2}$.

En la práctica clínica rutinaria, las mediciones con las formulas señaladas son parecidas para el mismo paciente; sin embargo, se ha observado variaciones significativas cuando la tasa de filtración glomerular es menor de $30 \mathrm{~mL} / \mathrm{min} / \mathrm{m}^{2}{ }^{(21)}$. Actualmente existe varias fórmulas que usan cistatina $C$ para calcular la tasa de filtración glomerular, algunos incluyen adicionalmente la creatinina sérica para mejorar su exactitud ${ }^{(24)}$.

\section{PASO 7. EVALUACIÓN E INTERVENCIÓN DE LA INFRAPRESCRIPCIÓN}

El fenómeno conocido como infraprescripción de fármacos es relativamente frecuente en pacientes geriátricos que son excluidos de intervenciones terapéuticas -sobre bases de edad únicamente- que han demostrado disminución de morbilidad y mortalidad (Ejem. anticoagulación o terapia antiplaquetaria en pacientes con fibrilación auricular aislada para la prevención de desórdenes cerebrovasculares embólicos).

Para evitar la infraprescripción, se recomienda el uso de los criterios START (Screening Tool to Alert doctors to Right Treatment) ${ }^{(15)}$, una herramienta útil que nos recuerda la prescripción de medicamentos a ser considerados en personas de 65 o más años, que tengan indicaciones o diagnóstico de enfermedades, consta de 22 criterios descritos como recomendaciones sobre inicio de una terapia farmacológica específica para cada diagnóstico realizado, si no existe una contraindicación para la intervención farmacológica (Ejem. "Debería prescribirse warfarina en pacientes mayores con fibrilación auricular si no existen contraindicaciones para la administración de este fármaco"). Como su acrónimo START (iniciar, comenzar) lo exige, estos criterios nos recuerdan las intervenciones terapéuticas a iniciar en pacientes geriátricos previa valoración geriátrica integral y evaluación individualizada del riesgo en contraste con el beneficio de la farmacoterapia.

\section{PASO 8. PREVENIR LAS REACCIONES ADVERSAS POR RETIRO INADECUADO DE FÁRMACOS}

Si se ha decidido suspender un medicamento determinado por diversas causas, antes de retirar dicho fármaco es importante verificar si este puede producir reacciones adversas por retiro inadecuado de medicamentos (RARM), es decir, signos y síntomas significativos causados por la suspensión de un fármaco ${ }^{(26)}$.

La sintomatología causada por una RARM puede ir desde la reaparición o el rebote de los trastornos para los cuales el fármaco fue administrado (Ejem. hipertensión después de suspender la terapia con clonidina), hasta la aparición de nuevos complejos sintomáticos (Ejem. debilidad y nauseas después de parar la terapia con corticoides en pacientes con enfermedad pulmonar obstructiva crónica).

Otro tipo de RARM es la exacerbación de la enfermedad subyacente (Ejem. empeoramiento de la angina de pecho después de la discontinuación de la terapia con nitratos) para lo cual se administró el medicamento originalmente.

Las reacciones fisiológicas por el retiro de fármacos pueden ocurrir dentro de las 2 semanas de discontinuación del fármaco (excepto para los betabloqueadores y benzodiazepinas, donde puede ocurrir hasta la cuarta semana) y empeoramiento de la enfermedad que puede ocurrir dentro de las 4 semanas de haber suspendido el medicamento.

Los principales medicamentos que pueden causar una RARM, a menos que se disminuya gradualmente, son los betabloqueadores, simpaticomiméticos de acción central, sedantes-hipnóticos, opiáceos, antidepresivos tricíclicos, antipsicóticos, estimulantes y corticosteroides (26). Un tópico muy importante relacionado con los RARM es su prevención en pacientes hospitalizados en los servicios de cirugía en el perioperatorio ${ }^{(27)}$.

\section{PASO 9. EVALUAR LA CAPACIDAD DE MANEJO DE MEDICAMENTOS DE LA PERSONA MAYOR}

La capacidad de manejo de medicamentos (CMM) en personas mayores se define como una actividad instrumental de la vida diaria (AIVD), que requiere la correcta coordinación de habilidades cognitivas y funcionales para llevar a cabo todos procesos implicados en la autoadministración de un medicamento prescrito ${ }^{(28)}$.

Los principales procesos, que abarcan desde la recepción de la receta médica hasta la administración del medicamento, pueden clasificarse de la siguiente manera: proceso de recepción de la receta médica; adquisición de los medicamentos; almacenamiento; preparación de la dosis, y administración o toma del fármaco ${ }^{(28)}$.

Para la medición de la CMM se han diseñado una serie de instrumentos, el que tiene el mejor perfil 
Tabla 1. Información mínima sobre el fármaco prescrito al paciente recomendada por la Organización Mundial de la Salud

\section{EFECTOS DEL MEDICAMENTO}

Por qué es necesario.

- Qué síntomas desaparecerán y cuáles no.

- Cuándo se espera que se inicie el efecto.

- Qué puede ocurrir si el medicamento se toma de manera incorrecta o no se toma.

2. EFECTOS INDESEADOS

- Cuáles pueden ocurrir.

- Cómo reconocerlos.

- Cuánto durarán.

- Su gravedad.

- Qué hacer.

\section{INSTRUCCIONES}

- Cómo se debe tomar el medicamento.

- Cuánto debe durar el tratamiento.

- Cómo se debe guardar el medicamento.

- Qué hacer con el medicamento sobrante.

\section{ADVERTENCIAS}

- Cuándo no se debe tomar el medicamento.

- Cuál es la dosis máxima.

- Por qué se debe tomar la tanda completa de tratamiento.

\section{PRÓXIMA CITA}

- Cuándo volver (o no).

- En qué circunstancias debe volver antes de lo previsto.

- Información que el médico necesitará en la próxima cita.

6. ¿ESTÁ TODO CLARO?

- Pregunte al paciente si ha entendido toda la explicación.

- Pídale al paciente que repita la información más importante.

- Pregúntele si tiene más preguntas.

Adaptado de: De Vries ThPGM, Henneing RH, Hogerzeil HV, Fresle DA. Guide to good prescribing. WHO/DAP/94.11. Geneva. World Health Organization, 1994

de rendimiento y es la más utilizado es la escala de medición de capacidad de auto-administración de medicamentos (Drug Regimen Unassisted Grading Scale o DRUGS) ${ }^{(29)}$.

El objetivo de la evaluación de la capacidad manejo medicamentos de la persona mayor, es poder observar si el paciente puede autoadministrarse el medicamento viviendo una vida independiente, si existiera dificultades en esta evaluación será necesario la intervención sobre los aspectos cognitivos, sensoriales o mecánicos para asegurar la adherencia a la medicación.

La existencia de serias dificultades en esta prueba es una indicación de la necesidad de un cuidador que vigile o ayude al cumplimiento de la farmacoterapia.

\section{PASO 10. HOJA DE INFORMACIÓN MÍNIMA AL PACIENTE}

El paciente no debe salir de alta, o de la consulta externa, sin recibir su hoja de información mínima sobre el fármaco prescrito, recomendada por la Organización Mundial de la Salud (30) (Tabla 1). En ella están contenidas todas las indicaciones para la toma correcta de la medicación administrada.

\section{CONCLUSIÓN}

La morbimortalidad inducida por el uso inadecuado de medicamentos es una realidad, sabemos que existen problemas de seguridad que son susceptibles de prevenir. Es tiempo de actuar y de aplicar lo aprendido con todas las estrategias necesarias para construir un sistema seguro y racional del uso de medicamentos en personas mayores.

La presente lista de chequeo, es una suma de instrumentos previamente validados para una estrategia global de uso seguro de los medicamentos en personas mayores, que puede ser de gran ayuda para sistematizar dicha tarea, ya sea en el contexto clínico ambulatorio u hospitalario.

Fuentes de financiamiento: autofinanciado.

Conflictos de interés: el autor declara no tener conflictos de interés.

\section{REFERENCIAS BIBLIOGRÁFICAS}

1. Kohn LT, Corrigan JM, Donaldson MS. To err is human. Building a safer health system. Washington, DC: National Academy Press; 1999.

2. Taché SV, Sönnichsen A, Ashcroft DM. Prevalence of adverse drug events in ambulatory care: a systematic review. 2011;45(7-8):977-89.

3. Budnitz DS, Lovegrove MC, Shehab N, Richards CL. Emergency hospitalizations for adverse drug events in older Americans. N Engl J Med. 2011;365(21):2002-12.

4. Bootman JL, Harrison DL, Cox E. The health care cost of drug-related morbidity and mortality in nursing facilities. Arch Inter Med. 1997;157(18):2089-96.

5. Oscanoa TJ. Diagnóstico de problemas relacionados con medicamentos en adultos mayores al momento de ser hospitalizados. Rev Peru Med Exp Salud Publica. 2011;28(2):256-63.
6. Spinewine A, Schmader KE, Barber $\mathrm{N}$, Hughes C, Lapane KL, Swine $\mathrm{C}$, et al. Appropriate prescribing in elderly people: how well can it be measured and optimised? Lancet 2007;370(9582):173-84.

7. Rochon PA, Gurwitz JH. Prescribing for seniors: neither too much nor too little. JAMA. 1999;282(2):113-5.

8. Liu CL, Peng LN, Chen YT, Lin MH, Liu LK, Chen LK. Potentially 
inappropriate prescribing (IP) for elderly medical inpatients in Taiwan: a hospital-based study. Arch Gerontol Geriatr. 2012;55(1):148-51.

9. Fick DM, Cooper JW, Wade WE, Waller JL, Maclean JR, Beers $\mathrm{MH}$. Updating the Beers criteria for potentially inappropriate medication use in older adults: results of a US consensus panel of experts. Arch Intern Med. 2003;163(22):2716-24.

10. American Geriatrics Society 2012 Beers Criteria Update Expert Panel. American Geriatrics Society updated Beers Criteria for potentially inappropriate medication use in older adults. J Am Geriatr Soc. 2012;60(4):616-31.

11. Jano E, Aparasu RR. Healthcare outcomes associated with beers' criteria: a systematic review. Ann Pharmacother. 2007;41(3):438-47.

12. Nathan A, Goodyer L, Lovejoy A, Rashid A. 'Brown bag' medication reviews as a means of optimizing patients' use of medication and of identifying potential clinical problems. Fam Pract. 1999;16(3):278-82.

13. Howell L, Kochhar K, Saywell R Jr, Zollinger T, Koehler J, Mandzuk $\mathrm{C}$, et al. Use of herbal remedies by Hispanic patients: do they inform their physician? J Am Board Fam Med. 2006;19(6):566-78.

14. Woolcott JC, Richardson KJ, Wiens MO, Patel B, Marin J, Khan KM, et al. Metaanalysis of the impact of 9 medication classes on falls in elderly persons. Arch Intern Med. 2009;169(21):1952-60.

15. Rudolph JL, Salow MJ, Angelini MC, McGlinchey RE. The anticholinergic risk scale and anticholinergic adverse effects in older persons. Arch Intern Med. 2008;168(5):508-13.
16. Hilmer SN, Mager DE, Simonsick EM, Cao Y, Ling SM, Windham BG, et al. A drug burden index to define the functional burden of medications in older people. Arch Intern Med. 2007;167(8):781-7.

17. Hanlon JT, Schmader KE, Samsa GP, Weinberger M, Uttech KM, Lewis IK, et al. A method for assessing drug therapy appropriateness. J Clin Epidemiol. 1992;45(10):1045-51.

18. Márquez Contreras E, Casado Martínez JJ, Márquez Cabeza JJ. Estrategias para mejorar el cumplimiento. FMC 2001;8(8):558-73.

19. Osterberg L, Blaschke T. Adherence to medication. N Engl J Med. 2005;353(5):487-97.

20. Morisky DE, Green LW, Levine DM. Concurrent and predictive validity of a self-reported measure of medication adherence. Med Care. 1986;24(1):6774.

21. Van Pottelbergh G, Van Heden L, Matheï C, Degryse J. Methods to evaluate renal function in elderly patients: a systematic literature review. Age Ageing. 2010;39(5):542-8.

22. Cockcroft DW, Gault MH. Prediction of creatinine clearance from serum creatinine. Nephron. 1976;16(1):31-41.

23. Levey AS, Bosch JP, Lewis JB, Greene T, Rogers N, Roth D. A more accurate method to estimate glomerular filtration rate from serum creatinine: a new prediction equation. Modification of Diet in Renal Disease Study Group. Ann Intern Med. 1999;130(6):461-70.

24. Inker LA, Schmid CH, Tighiouart $\mathrm{H}$, Eckfeldt JH, Feldman HI, Greene T, et al. Estimating glomerular filtration rate from serum creatinine and cystatin C. N Engl J Med. 2012;367(1):20-9.
25. Gallagher P, Ryan C, Byrne S, Kennedy J, O’Mahony D. STOPP (Screening Tool of Older Person's Prescriptions) and START (Screening Tool to Alert doctors to Right Treatment). Consensus validation. Int J Clin Pharm Ther. 2008;46(2):72-83.

26. Graves T, Hanlon JT, Schmader KE, Landsman PB, Samsa GP, Pieper CF, et al. Adverse events after discontinuing medications in elderly outpatients. Arch Intern Med. 1997;157(19):220510.

27. Spell NO 3rd. Stopping and restarting medications in the perioperative period. Med Clin N Am. 2001;85(5):111728.

28. Beckman Gyllenstrand A. Medication management and patient compliance in old age [Internet]. Thesis for Doctoral Grade. Karolinska Institute, Stockholm, Sweden. 2007 [citado el 31 diciembre 2010]. Disponible en: http://diss.kib. ki.se/2007/978-91-7357-166-1/thesis. pdf

29. Edelberg HK, Shallenberger E, Wei JY: Medication management capacity in highly functioning community-living older adults: detection of early deficits. J Am Geriatr Soc. 1999;47(5):592-6.

30. De Vries ThPGM, Henneing RH, Hogerzeil HV, Fresle DA. Guide to good prescribing. A practical manual. WHO/DAP/94.11. Geneva: World Health Organization; 1994.

Correspondencia: Teodoro Oscanoa Espinoza Dirección: Hospital Almenara. Av. Grau 800. Lima 13. Perú.

Teléfono: 3242983 anexo 4085

Correo electrónico: tjoscanoae@gmail.com

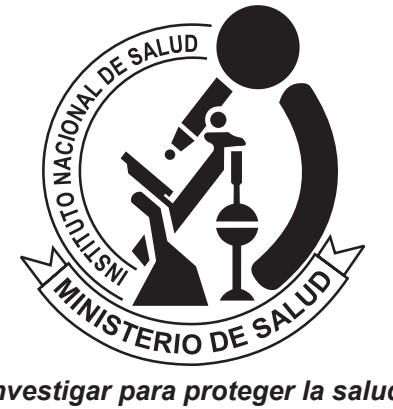

Investigar para proteger la salud
REVISTA PERUANA DE MEDICINA

EXPERIMENTAL Y SALUD PÚBLICA

CUMPLIENDO SUS METAS Y

PROYECTÁNDOSE AL FUTURO

\section{Visite los contenidos de la revista en: www.ins.gob.pe/rpmesp}

